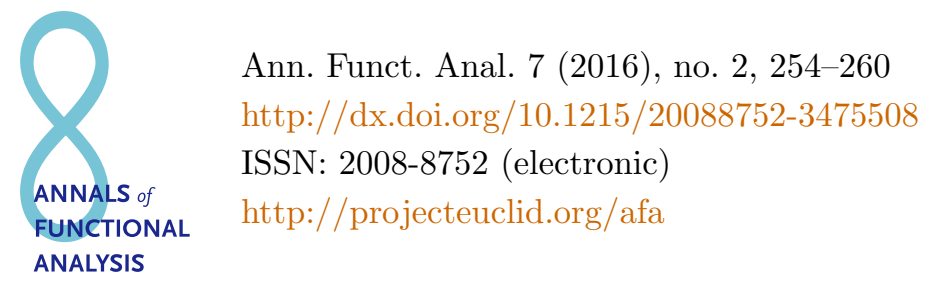

\title{
ADDITIVE MAPS PRESERVING THE SEMI-FREDHOLM DOMAIN IN SPECTRUM
}

\author{
WEIJUAN SHI ${ }^{1}$ and GUOXING $\mathrm{JI}^{2^{*}}$ \\ Communicated by M. Ptak
}

\begin{abstract}
Let $\mathcal{X}$ be an infinite-dimensional complex Banach space and let $\mathcal{B}(\mathcal{X})$ be the algebra of all bounded linear operators. In this article we show that an additive surjective map $\varphi$ on $\mathcal{B}(\mathcal{X})$ preserving the semi-Fredholm domain in spectrum is an automorphism or an antiautomorphism on $\mathcal{B}(\mathcal{X})$.
\end{abstract}

\section{INTRODUCTION}

Preserver problems aim to characterize those linear or nonlinear maps on operator algebras preserving certain properties, subsets, or relations. One of the most famous problems in this direction is Kaplansky's problem (see [6]) asking whether every surjective unital invertibility-preserving linear map between two semisimple Banach algebras is a Jordan homomorphism. This problem was first solved in the finite-dimensional case. J. Dieudonné in [4] and Marcus and Purves in [7] proved that every unital invertibility-preserving linear map on a complex matrix algebra is either an inner automorphism or an inner antiautomorphism. This result was later extended to the algebra of all bounded linear operators on a Banach space by A. R. Sourour in [10] and to von Neumann algebras by B. Aupetit in [1]. As we know, spectrum is a very fundamental and key concept in operator theory. Hence many authors (see [1], [3], [5]) are interested in preserver problems related to the spectrum as well as to certain parts of the spectrum. For example, in [3], Cui and Hou showed that additive maps on standard operator algebras preserving parts of the spectrum are either isomorphisms or anti-isomorphisms. It is known

Copyright 2016 by the Tusi Mathematical Research Group.

Received Mar. 12, 2015; Accepted Jun. 29, 2015.

${ }^{*}$ Corresponding author.

2010 Mathematics Subject Classification. Primary 47B48; Secondary 47A10.

Keywords. semi-Fredholm domain, additive map, additive preserver. 


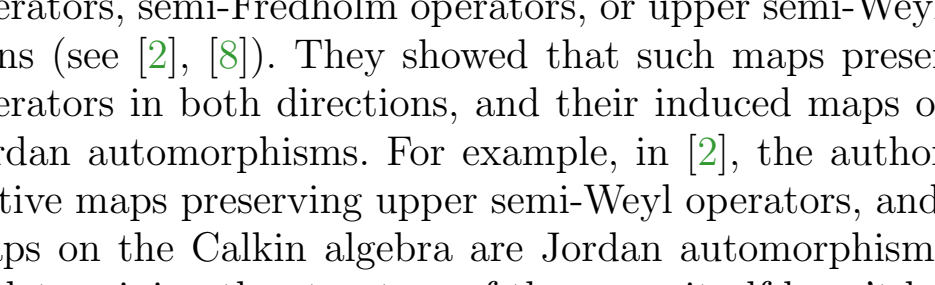

\title{
ADDITIVE MAPS PRESERVING THE SEMI-FREDHOLM DOMAIN IN SPECTRUM
}

\author{
WEIJUAN SHI ${ }^{1}$ and GUOXING $\mathrm{JI}^{2^{*}}$ \\ Communicated by M. Ptak
}

\begin{abstract}
Let $\mathcal{X}$ be an infinite-dimensional complex Banach space and let $\mathcal{B}(\mathcal{X})$ be the algebra of all bounded linear operators. In this article we show that an additive surjective map $\varphi$ on $\mathcal{B}(\mathcal{X})$ preserving the semi-Fredholm domain in spectrum is an automorphism or an antiautomorphism on $\mathcal{B}(\mathcal{X})$.
\end{abstract}

\section{INTRODUCTION}

Preserver problems aim to characterize those linear or nonlinear maps on operator algebras preserving certain properties, subsets, or relations. One of the most famous problems in this direction is Kaplansky's problem (see [6]) asking whether every surjective unital invertibility-preserving linear map between two semisimple Banach algebras is a Jordan homomorphism. This problem was first solved in the finite-dimensional case. J. Dieudonné in [4] and Marcus and Purves in [7] proved that every unital invertibility-preserving linear map on a complex matrix algebra is either an inner automorphism or an inner antiautomorphism. This result was later extended to the algebra of all bounded linear operators on a Banach space by A. R. Sourour in [10] and to von Neumann algebras by B. Aupetit in [1]. As we know, spectrum is a very fundamental and key concept in operator theory. Hence many authors (see [1], [3], [5]) are interested in preserver problems related to the spectrum as well as to certain parts of the spectrum. For example, in [3], Cui and Hou showed that additive maps on standard operator algebras preserving parts of the spectrum are either isomorphisms or anti-isomorphisms. It is known

Copyright 2016 by the Tusi Mathematical Research Group.

Received Mar. 12, 2015; Accepted Jun. 29, 2015.

${ }^{*}$ Corresponding author.

2010 Mathematics Subject Classification. Primary 47B48; Secondary 47A10.

Keywords. semi-Fredholm domain, additive map, additive preserver. 


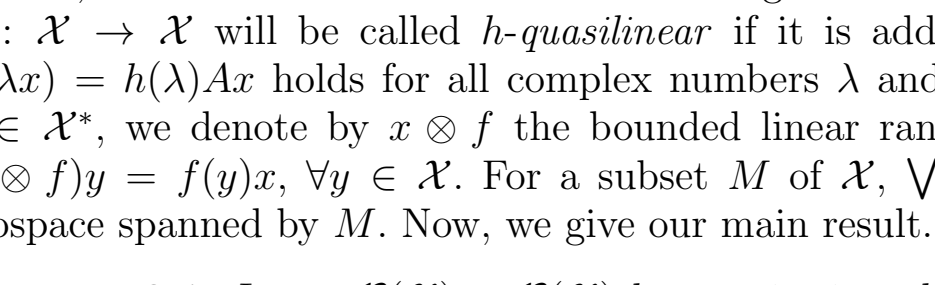

\title{
ADDITIVE MAPS PRESERVING THE SEMI-FREDHOLM DOMAIN IN SPECTRUM
}

\author{
WEIJUAN SHI ${ }^{1}$ and GUOXING $\mathrm{JI}^{2^{*}}$ \\ Communicated by M. Ptak
}

\begin{abstract}
Let $\mathcal{X}$ be an infinite-dimensional complex Banach space and let $\mathcal{B}(\mathcal{X})$ be the algebra of all bounded linear operators. In this article we show that an additive surjective map $\varphi$ on $\mathcal{B}(\mathcal{X})$ preserving the semi-Fredholm domain in spectrum is an automorphism or an antiautomorphism on $\mathcal{B}(\mathcal{X})$.
\end{abstract}

\section{INTRODUCTION}

Preserver problems aim to characterize those linear or nonlinear maps on operator algebras preserving certain properties, subsets, or relations. One of the most famous problems in this direction is Kaplansky's problem (see [6]) asking whether every surjective unital invertibility-preserving linear map between two semisimple Banach algebras is a Jordan homomorphism. This problem was first solved in the finite-dimensional case. J. Dieudonné in [4] and Marcus and Purves in [7] proved that every unital invertibility-preserving linear map on a complex matrix algebra is either an inner automorphism or an inner antiautomorphism. This result was later extended to the algebra of all bounded linear operators on a Banach space by A. R. Sourour in [10] and to von Neumann algebras by B. Aupetit in [1]. As we know, spectrum is a very fundamental and key concept in operator theory. Hence many authors (see [1], [3], [5]) are interested in preserver problems related to the spectrum as well as to certain parts of the spectrum. For example, in [3], Cui and Hou showed that additive maps on standard operator algebras preserving parts of the spectrum are either isomorphisms or anti-isomorphisms. It is known

Copyright 2016 by the Tusi Mathematical Research Group.

Received Mar. 12, 2015; Accepted Jun. 29, 2015.

${ }^{*}$ Corresponding author.

2010 Mathematics Subject Classification. Primary 47B48; Secondary 47A10.

Keywords. semi-Fredholm domain, additive map, additive preserver. 


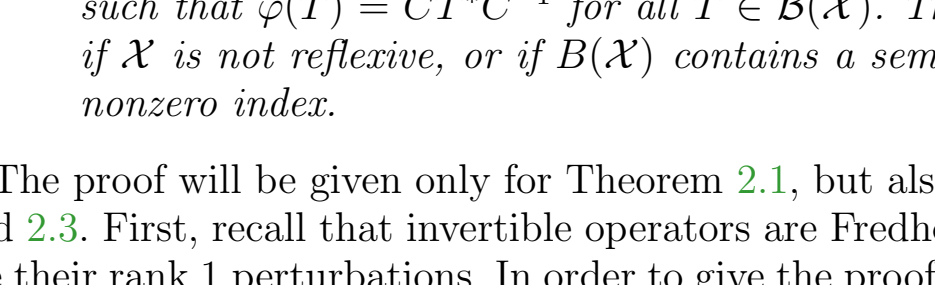

\title{
ADDITIVE MAPS PRESERVING THE SEMI-FREDHOLM DOMAIN IN SPECTRUM
}

\author{
WEIJUAN SHI ${ }^{1}$ and GUOXING $\mathrm{JI}^{2^{*}}$ \\ Communicated by M. Ptak
}

\begin{abstract}
Let $\mathcal{X}$ be an infinite-dimensional complex Banach space and let $\mathcal{B}(\mathcal{X})$ be the algebra of all bounded linear operators. In this article we show that an additive surjective map $\varphi$ on $\mathcal{B}(\mathcal{X})$ preserving the semi-Fredholm domain in spectrum is an automorphism or an antiautomorphism on $\mathcal{B}(\mathcal{X})$.
\end{abstract}

\section{INTRODUCTION}

Preserver problems aim to characterize those linear or nonlinear maps on operator algebras preserving certain properties, subsets, or relations. One of the most famous problems in this direction is Kaplansky's problem (see [6]) asking whether every surjective unital invertibility-preserving linear map between two semisimple Banach algebras is a Jordan homomorphism. This problem was first solved in the finite-dimensional case. J. Dieudonné in [4] and Marcus and Purves in [7] proved that every unital invertibility-preserving linear map on a complex matrix algebra is either an inner automorphism or an inner antiautomorphism. This result was later extended to the algebra of all bounded linear operators on a Banach space by A. R. Sourour in [10] and to von Neumann algebras by B. Aupetit in [1]. As we know, spectrum is a very fundamental and key concept in operator theory. Hence many authors (see [1], [3], [5]) are interested in preserver problems related to the spectrum as well as to certain parts of the spectrum. For example, in [3], Cui and Hou showed that additive maps on standard operator algebras preserving parts of the spectrum are either isomorphisms or anti-isomorphisms. It is known

Copyright 2016 by the Tusi Mathematical Research Group.

Received Mar. 12, 2015; Accepted Jun. 29, 2015.

${ }^{*}$ Corresponding author.

2010 Mathematics Subject Classification. Primary 47B48; Secondary 47A10.

Keywords. semi-Fredholm domain, additive map, additive preserver. 


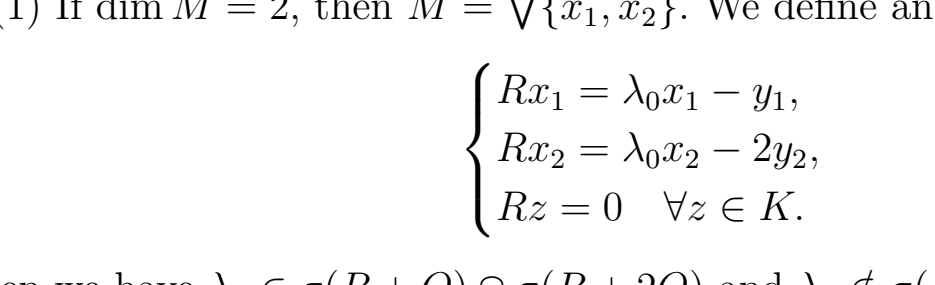

\title{
ADDITIVE MAPS PRESERVING THE SEMI-FREDHOLM DOMAIN IN SPECTRUM
}

\author{
WEIJUAN SHI ${ }^{1}$ and GUOXING $\mathrm{JI}^{2^{*}}$ \\ Communicated by M. Ptak
}

\begin{abstract}
Let $\mathcal{X}$ be an infinite-dimensional complex Banach space and let $\mathcal{B}(\mathcal{X})$ be the algebra of all bounded linear operators. In this article we show that an additive surjective map $\varphi$ on $\mathcal{B}(\mathcal{X})$ preserving the semi-Fredholm domain in spectrum is an automorphism or an antiautomorphism on $\mathcal{B}(\mathcal{X})$.
\end{abstract}

\section{INTRODUCTION}

Preserver problems aim to characterize those linear or nonlinear maps on operator algebras preserving certain properties, subsets, or relations. One of the most famous problems in this direction is Kaplansky's problem (see [6]) asking whether every surjective unital invertibility-preserving linear map between two semisimple Banach algebras is a Jordan homomorphism. This problem was first solved in the finite-dimensional case. J. Dieudonné in [4] and Marcus and Purves in [7] proved that every unital invertibility-preserving linear map on a complex matrix algebra is either an inner automorphism or an inner antiautomorphism. This result was later extended to the algebra of all bounded linear operators on a Banach space by A. R. Sourour in [10] and to von Neumann algebras by B. Aupetit in [1]. As we know, spectrum is a very fundamental and key concept in operator theory. Hence many authors (see [1], [3], [5]) are interested in preserver problems related to the spectrum as well as to certain parts of the spectrum. For example, in [3], Cui and Hou showed that additive maps on standard operator algebras preserving parts of the spectrum are either isomorphisms or anti-isomorphisms. It is known

Copyright 2016 by the Tusi Mathematical Research Group.

Received Mar. 12, 2015; Accepted Jun. 29, 2015.

${ }^{*}$ Corresponding author.

2010 Mathematics Subject Classification. Primary 47B48; Secondary 47A10.

Keywords. semi-Fredholm domain, additive map, additive preserver. 


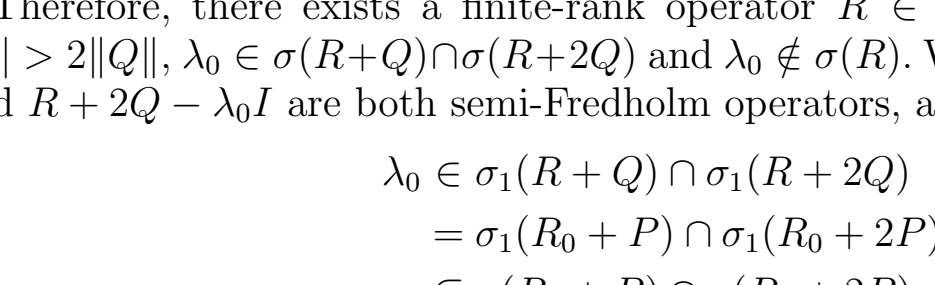

\title{
ADDITIVE MAPS PRESERVING THE SEMI-FREDHOLM DOMAIN IN SPECTRUM
}

\author{
WEIJUAN SHI ${ }^{1}$ and GUOXING $\mathrm{JI}^{2^{*}}$ \\ Communicated by M. Ptak
}

\begin{abstract}
Let $\mathcal{X}$ be an infinite-dimensional complex Banach space and let $\mathcal{B}(\mathcal{X})$ be the algebra of all bounded linear operators. In this article we show that an additive surjective map $\varphi$ on $\mathcal{B}(\mathcal{X})$ preserving the semi-Fredholm domain in spectrum is an automorphism or an antiautomorphism on $\mathcal{B}(\mathcal{X})$.
\end{abstract}

\section{INTRODUCTION}

Preserver problems aim to characterize those linear or nonlinear maps on operator algebras preserving certain properties, subsets, or relations. One of the most famous problems in this direction is Kaplansky's problem (see [6]) asking whether every surjective unital invertibility-preserving linear map between two semisimple Banach algebras is a Jordan homomorphism. This problem was first solved in the finite-dimensional case. J. Dieudonné in [4] and Marcus and Purves in [7] proved that every unital invertibility-preserving linear map on a complex matrix algebra is either an inner automorphism or an inner antiautomorphism. This result was later extended to the algebra of all bounded linear operators on a Banach space by A. R. Sourour in [10] and to von Neumann algebras by B. Aupetit in [1]. As we know, spectrum is a very fundamental and key concept in operator theory. Hence many authors (see [1], [3], [5]) are interested in preserver problems related to the spectrum as well as to certain parts of the spectrum. For example, in [3], Cui and Hou showed that additive maps on standard operator algebras preserving parts of the spectrum are either isomorphisms or anti-isomorphisms. It is known

Copyright 2016 by the Tusi Mathematical Research Group.

Received Mar. 12, 2015; Accepted Jun. 29, 2015.

${ }^{*}$ Corresponding author.

2010 Mathematics Subject Classification. Primary 47B48; Secondary 47A10.

Keywords. semi-Fredholm domain, additive map, additive preserver. 


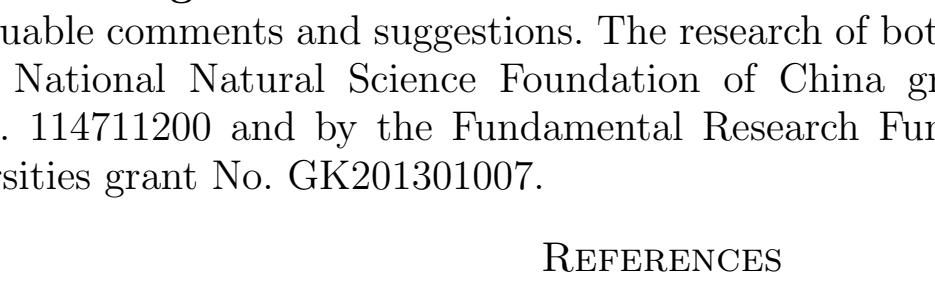

\title{
ADDITIVE MAPS PRESERVING THE SEMI-FREDHOLM DOMAIN IN SPECTRUM
}

\author{
WEIJUAN SHI ${ }^{1}$ and GUOXING $\mathrm{JI}^{2^{*}}$ \\ Communicated by M. Ptak
}

\begin{abstract}
Let $\mathcal{X}$ be an infinite-dimensional complex Banach space and let $\mathcal{B}(\mathcal{X})$ be the algebra of all bounded linear operators. In this article we show that an additive surjective map $\varphi$ on $\mathcal{B}(\mathcal{X})$ preserving the semi-Fredholm domain in spectrum is an automorphism or an antiautomorphism on $\mathcal{B}(\mathcal{X})$.
\end{abstract}

\section{INTRODUCTION}

Preserver problems aim to characterize those linear or nonlinear maps on operator algebras preserving certain properties, subsets, or relations. One of the most famous problems in this direction is Kaplansky's problem (see [6]) asking whether every surjective unital invertibility-preserving linear map between two semisimple Banach algebras is a Jordan homomorphism. This problem was first solved in the finite-dimensional case. J. Dieudonné in [4] and Marcus and Purves in [7] proved that every unital invertibility-preserving linear map on a complex matrix algebra is either an inner automorphism or an inner antiautomorphism. This result was later extended to the algebra of all bounded linear operators on a Banach space by A. R. Sourour in [10] and to von Neumann algebras by B. Aupetit in [1]. As we know, spectrum is a very fundamental and key concept in operator theory. Hence many authors (see [1], [3], [5]) are interested in preserver problems related to the spectrum as well as to certain parts of the spectrum. For example, in [3], Cui and Hou showed that additive maps on standard operator algebras preserving parts of the spectrum are either isomorphisms or anti-isomorphisms. It is known

Copyright 2016 by the Tusi Mathematical Research Group.

Received Mar. 12, 2015; Accepted Jun. 29, 2015.

${ }^{*}$ Corresponding author.

2010 Mathematics Subject Classification. Primary 47B48; Secondary 47A10.

Keywords. semi-Fredholm domain, additive map, additive preserver. 